\title{
REVIEW
}

\section{Two steps forward in bedside monitoring of lung mechanics: transpulmonary pressure and lung volume}

\author{
Gustavo A Cortes, John J Marini* \\ This article is one of ten reviews selected from the Annual Update in Intensive Care and Emergency Medicine 2013 and co-published as a series \\ in Critical Care. Other articles in the series can be found online at http://ccforum.com/series/annualupdate2013. Further information about the \\ Annual Update in Intensive Care and Emergency Medicine is available from http://www.springer.com/series/8901.
}

\section{Introduction}

For many decades, pressure-based respiratory mechanics have served to aid the judgment of clinicians when monitoring mechanical ventilation and making important decisions in respiratory care. However, measurements based on airway pressure $\left(\mathrm{P}_{\mathrm{AW}}\right)$ alone have limited ability to generate individualized insights for a diverse patient population with varied pathologic conditions. While the passive lungs are the primary target of attention, $\mathrm{P}_{\mathrm{AW}^{-}}$ based interpretations may be influenced by differences in breathing pattern, alterations in chest wall activity (including diaphragmatic function), changes in lung volume, asymmetry of lung disease, abdominal distension, etc. All of these factors may complicate the interpretation of respiratory mechanics and make fixed criteria for safe ventilation difficult to apply.

Functional residual capacity (FRC) and calculated transpulmonary pressure $\left(\mathrm{P}_{\mathrm{TP}}\right)$ are two components of the bedside monitoring array recently introduced into clinical practice. Used separately, and together, they complement and may improve interpretations stemming from parameters of lung mechanics based on $\mathrm{P}_{\mathrm{AW}}$ alone. As a more physiologic approach, monitoring FRC and $\mathrm{P}_{\mathrm{TP}}$ represents an opportunity to individualize the interpretation of lung mechanics and guide development of a ventilator strategy tailored to the specifics of a given patient.

In this update, we briefly address the management rationale and technical background for monitoring FRC and calculating $\mathrm{P}_{\mathrm{TP}}$ placing major emphasis on the potential clinical applicability of these two missing pieces in bedside monitoring.

*Correspondence: John.J.Marini@HealthPartners.com

Department of Pulmonary and Critical Care, University of Minnesota, Regions

Hospital, 640 Jackson Street St. Paul, MN 55101, USA

\section{Esophageal pressure and calculated transpulmonary pressure}

$\mathrm{P}_{\text {TP }}$ (alveolar pressure - esophageal pressure [Pes]) is a conceptual step closer to what is actually needed for monitoring when the object of interest is the lung itself. Pes has been used in the physiology laboratory to estimate pleural pressure for more than five decades $[1,2]$. Balloon catheter systems have been shown to be both precise and practical in measuring local Pes [3]. The small quantity of gas within the balloon tends to concentrate where the pressure of the surrounding tissue is most negative. A series of holes in the catheter, arranged in a spiral pattern along a $10 \mathrm{~cm}$ length, transmits the most negative pressure surrounding the catheter in a given horizontal plane [4]. Using this system implies that important logistical aspects, such as positioning of the esophageal balloon catheter [5], amount of insufflated gas and compliance of the balloon, have been addressed so as to promote fidelity of the Pes measurements [1].

Baydur's technique for placing the esophageal balloon [5] has been tested and found to be generally valid in spontaneously breathing subjects in sitting, supine, and lateral positions. This maneuver is conducted by occluding the airways at end-expiration and measuring the ratio of changes in esophageal and airway pressure during spontaneous inspiratory efforts made during occlusion. With lung volume unchanging, the fluctuations of both esophageal and airway pressure should be theoretically equivalent [5]. In subjects who are not spontaneously breathing, however, other cues and feedbacks must be used to assure appropriate positioning of the catheter that senses esophageal pressure. The technique used by Talmor, Loring and colleagues in passively ventilated patients [6] involved advancing the catheter into the stomach as a first step. This initial location was 
verified by transiently increasing balloon pressure with abdominal compression. Subsequently, the catheter was withdrawn into the esophagus, using obvious cardiac oscillations and changes in $\mathrm{P}_{\mathrm{TP}}$ during tidal ventilation to adjust the esophageal balloon catheter to the correct position [6]. This method may reduce the technical challenges accompanying placement in the clinical setting during passive mechanical ventilation without affecting catheter reliability.

\section{Interpreting Pes measurements: what is Pes really measuring?}

According to observations made by Agostoni et al. [7-9], tidal changes in Pes correlate with those of the pleural pressure applied to the surface of the lung, thereby enabling a valid estimation of $P_{T P}$ based on the difference between estimated alveolar pressure and Pes [7-9]. However, the pressure vector generated by the weight of mediastinal content (mediastinal artifact) may increase Pes in the supine position [10]. Additionally, Pes represents the least positive local pressure along its own horizontal (gravitational) plane in the upright position [4]; even with position unchanged, absolute $\mathrm{P}_{\mathrm{TP}}$ values elsewhere in the chest are theoretically different. For such reasons, the ability of Pes to track global average changes in pleural pressure may be limited when supine and in the presence of asymmetrical lung disease [11].

Absolute values of Pes are not only influenced by the 'mediastinal artifact' as a result of re-positioning from sitting to supine, but also by elevation of intra-abdominal pressure (IAP) and position-related lung volume changes. Recently, Owens et al. [12] concluded that Pes measurement artifacts imposed by mediastinal weight and postural effects are within a clinically acceptable range. These authors [12] compared the changes in end-expiratory Pes secondary to position changes in a cohort of overweight/obese spontaneously breathing patients with those occurring in lean subjects. Despite sitting and supine end-expiratory Pes values that were higher in the overweight/obese cohort than in the lean cohort, the observed changes in end-expiratory Pes as a result of repositioning from sitting to supine were unexpectedly similar in both groups [12]. These results point toward a relatively constant increment in Pes attributable to 'mediastinal artifact' when supine, independent of the body mass index (BMI). Moreover, elevated IAP and reduced chest wall compliance appear to explain components of the higher end-expiratory Pes values encountered in both positions among overweight/obese subjects [12].

After all these considerations, it might be concluded that despite strong studies supporting Pes as a reliable surrogate for pleural pressure [1-3], esophageal balloon estimation of pleural pressure may be influenced by regional characteristics of the sampled horizontal plane when supine, and also by pulmonary and/or extrapulmonary conditions, such as elevated IAP, obesity, and heterogeneity of lung disease [13,14]. Whatever the shortcomings of esophageal manometry may be, the reported data support its reliability in sampling a local region surrounding the lung when supine - the potentially critical and clinically relevant dependent zone.

\section{The role of transpulmonary pressure in acute lung injury}

Acute lung injury (ALI) and the acute respiratory distress syndrome (ARDS) are challenging respiratory conditions that require careful tuning of mechanical ventilation settings to improve oxygenation without inflicting injury [15]. To achieve adequate physiologic goals and simultaneously prevent ventilator-induced lung injury (VILI), transpulmonary pressure monitoring has been proposed as a promising approach to guide ventilation strategy in ALI/ARDS settings [6]. Airway pressure-based plateau and positive end-expiratory pressure (PEEP) values are simply not enough.

An influential study already mentioned [6] evaluated the value of monitoring Pes and calculating $\mathrm{P}_{\mathrm{TP}}$ in order to find a level of PEEP that could maintain oxygenation while theoretically preventing lung injury secondary to alveolar collapse or overdistension in patients with ALI/ ARDS [6]. Patients in the "esophageal pressure-guided group" underwent mechanical ventilation with PEEP adjusted by Pes measurements and $\mathrm{P}_{\mathrm{TP}}$ calculations; the "control group" of patients was mechanically ventilated according to the ARDS Network (ARDSnet) recommendations [15]. PEEP levels were adjusted to achieve an end-expiratory $\mathrm{P}_{\mathrm{TP}}$ within a positive range of 0 $10 \mathrm{~cm} \mathrm{H}_{2} \mathrm{O}$ and tidal volume was limited to keep endinspiratory $\mathrm{P}_{\mathrm{TP}}<25 \mathrm{~cm} \mathrm{H}_{2} \mathrm{O}$ - a threshold never encountered in any of the studied patients. At 72 hours, the patients in the "esophageal pressure-guided group" had a ratio of partial pressure of arterial oxygen to the fraction of inspired oxygen $\left(\mathrm{PaO}_{2} / \mathrm{FiO}_{2}\right)$ which averaged $88 \mathrm{~mm} \mathrm{Hg}$ higher than in the control group. Similarly, improvement in respiratory-system compliance was also observed in the "esophageal pressure-guided group". However, despite trends toward improved survival, this study does not provide uncontestable data supporting reduction in mortality associated with this mechanical ventilation strategy guided by $\mathrm{P}_{\mathrm{TP}}$ estimations in patients with ALI/ ARDS [6].

Other studies suggest Pes measurement as a physiologically defensible and reliable tool for estimating $\mathrm{P}_{\mathrm{TP}}$ in critically ill patients $[13,14,16]$. As an example of such enthusiastic reports, Grasso et al. [16] evaluated whether keeping end-inspiratory $\mathrm{P}_{\mathrm{TP}}$ within a theoretically innocuous range might allow safe increases of PEEP in pursuit of improved oxygenation. These authors found that relaxing the excessively prudent $\mathrm{P}_{\mathrm{Aw}}$-based criteria for 
safe ventilation (justified by $\mathrm{P}_{\text {TP }}$ calculations) may avoid unnecessary use of extracorporeal membrane oxygenation (ECMO) in patients with ALI/ARDS from influenza A (H1N1) infection [16]. Although such data are encouraging, we believe that although clinically feasible, measuring $\mathrm{P}_{\mathrm{ES}}$ and calculating $\mathrm{P}_{\mathrm{TP}}$ as a strategy for setting the parameters of ventilator support must be embraced with caution, especially in the setting of lung injury, where the tension of the alveolar microenvironment may only be roughly represented by the $\mathrm{P}_{\mathrm{TP}}$. Additionally, increasing PEEP using $\mathrm{P}_{\text {TP }}$ monitoring might be consider 'safe' with regard to the mechanics of pulmonary injury, but may be simultaneously associated with hazardous consequences for hemodynamics [17].

The degree to which Pes is influenced by positioning, abdominal distension, spontaneously breathing efforts, and other conditions associated with reduced chest wall compliance in the setting of ALI/ARDS is still unclear and requires further clinical investigation. For example, estimates of $\mathrm{P}_{\mathrm{TP}}$ based on Pes measurements are almost certain to imprecisely represent all stresses within an asymmetrically compromised lung [11]. The volumealtering effects of unilateral pleural effusion were radically different for the two lungs of experimental animals, and yet the calculated $\mathrm{P}_{\mathrm{TP}}$ was little affected by fluid instillation [11]. In other words, we cannot expect a single local pressure to represent stresses everywhere across the topography of a heterogeneous thorax.

Nonetheless, $\mathrm{P}_{\mathrm{TP}}$ monitoring deserves credit for shifting the attention of clinicians to a more individually-tailored physiologic understanding of the respiratory function changes that occur during ALI and, although not perfect, estimations of $\mathrm{P}_{\mathrm{TP}}$ are of more help in elucidating the interactions between patient characteristics, disease conditions and ventilator settings than are pulmonary mechanics based on airway pressure alone [17] (Table 1).

\section{Monitoring functional residual capacity}

Measuring absolute resting gas volume (FRC) is an essential component of the testing battery needed to interpret lung mechanics in the out-patient pulmonary function laboratory. However, the measurement of FRC in the ventilated patient has faced logistical and technical challenges [18]. In a dynamic process of evolution, attempts to monitor FRC that were based on body plethysmography and planimetry (X-ray quantification method) [19] have now migrated to more sophisticated ventilator-integrated systems. These newer methods allow bedside measurement of FRC without interrupting mechanical ventilation, making accurate FRC measurement feasible in the critically ill [20]. Conceptually, FRC provides information to the clinician that cannot be easily inferred from the $\mathrm{P}_{\mathrm{Aw}}$, tidal flow, and volume data available to this point in time.
Technological development of serial FRC testing

a. Equilibration method

Gas-dilution methods for quantifying FRC were developed as early as 1800 using inspired hydrogen [21]. Closer to the present day, helium gas equilibration methods have been used for research in patients. Such techniques involve manual (bag) ventilation after disconnection of the endotracheal tube from the mechanical ventilator, so that a fixed volume and concentration of helium is uniformly distributed between the lungs and bag after approximately 10 breaths taken through a closed circuit. The proportion of helium remaining in the bag after the equilibration period provides a direct dilution estimate of FRC, which has been reported accurate when compared to other methods [22]. This method requires interruption of care to connect the tracer gas, thereby increasing the risk of lung de-recruitment and cannot be conducted in unstable patients. Moreover, accuracy depends on the timing and skill of the operator conducting the measurement.

\section{b. Wash-out methods}

When tracer gas is added to or washed from the lungs during ventilation with serial fixed tidal volumes, the rate of change to the new concentration relates inversely to FRC. With this rationale, a method for estimating FRC from the wash-in/wash-out rate of a 'tracking' gas was first described by Durig in 1903 [23] and then by Darling et al. in 1940 [24]. Variants of such methods have used changing concentrations of sulfur hexafluoride (SF6), oxygen $\left(\mathrm{O}_{2}\right)$ and/or nitrogen $\left(\mathrm{N}_{2}\right)$. In 1993, Fretschner et al. [25] measured FRC via integrated nitrogen wash-in/out in a test lung model and in ventilated patients exposed to $\mathrm{FiO}_{2}$ changes of 0.3 - a method that involved intra-breath signal synchronization of flow and $\mathrm{FiO}_{2}$. This innovation allowed for the determination of FRC values without ventilator disconnection, but incurred an error of approximately $20 \%$ [25].

Recently, intricate and rapidly responding sensors have used sampling of respired gases from the ventilator circuitry to calculate FRC more safely and with relative accuracy without the need to interrupt ventilation. Gas-automated FRC measurement has been improved by using precise solenoid control and software synchronization of signals (flow and gas concentration) during ventilation. One example (Engstrom Carestation $^{\oplus}$ technology, GE Healthcare Madison, WI) of this approach directly measures the end-expiratory lung volume by slightly altering the delivered $\mathrm{FiO}_{2}$ level (step changes of only 0.1 ) for short periods of time using its volumetric $\mathrm{O}_{2}$ and $\mathrm{CO}_{2}$ measurement capability [26]. In a previous study we compared this method with ('gold standard') quantitative computer 
Table 1. Potential added values of two newly available tools in bedside monitoring*

\begin{tabular}{ll}
\hline Transpulmonary pressure & Functional residual capacity \\
\hline $\begin{array}{l}\text { Non-invasively estimates lung-distending pressure } \\
\text { Samples 'local' (but potentially critical) dependent zones of the lungs }\end{array}$ & $\begin{array}{l}\text { Non-invasively estimates 'functional' lung size, which refines compliance and } \\
\text { resistance calculations }\end{array}$ \\
Presents a new approach for setting 'effective' PEEP level in ALI/ARDS patients & Helps monitor disease progression and resolution \\
Offers the potential for monitoring 'true' driving pressures across the lung & $\begin{array}{l}\text { Allows assessment of the impact of common interventions: PEEP and } \\
\text { positioning } \\
\text { (tidal changes in } P_{\text {TP }} \text { ), despite abnormalities of the chest wall and effort }\end{array}$ \\
& $\begin{array}{l}\text { Complements } P_{\text {TP }} \text { in the setting of non-symmetrical lung and chest wall } \\
\text { diseases (e.g., atelectasis, effusion, etc.) }\end{array}$ \\
& $\begin{array}{l}\text { Optimizes lung-protection strategy by determining the size of the 'baby lung', } \\
\text { allowing for calculation of the'strain ratio' }\end{array}$ \\
\hline
\end{tabular}

*see text for limitations. ALI: Acute lung injury; ARDS: Acute respiratory distress syndrome; $\mathrm{P}_{\mathrm{TP}:}$ Transpulmonary pressure; PEEP: Positive end-expiratory pressure.

tomographic (CT) imaging and found that this automated method correlated well (across a wide range of end-expiratory lung volumes) [20].

\section{c. Technical limitations}

Some limitations, however, must be acknowledged regarding the measurement of FRC by gas washout in clinical practice. For example, rapid and/or irregular respiratory rates with large variations in tidal volume may alter FRC values and/or prevent gas-automated methods from performing the measurement [27]. Abnormal metabolic states because of high fever and/ or agitation, as well as neurological conditions that alter respiration may also influence FRC measurements by varying $\mathrm{CO}_{2}$ production and breathing patterns $[27,28]$.

\section{Rationale for monitoring FRC in the critical care setting}

FRC has been studied in ventilated patients for more than twenty years [29]. The effect of PEEP on FRC has been assessed and quantified by many investigators [3032], whose work taken together has concluded that PEEP invariably increases FRC determined by gas dilution methods, according to the well known pressure-volume (P-V) relationship of the respiratory system [30-32]. In one study, this incremental effect of PEEP was observed with normal lungs, primary lung disease, and secondary lung disease for PEEP values up to $15 \mathrm{~cm} \mathrm{H}_{2} \mathrm{O}$; FRC increased in proportion to the applied PEEP increments [30].

The FRC measured in response to PEEP admixes volumes resulting from recruitment of reopened units and expansion of the already patent ones. Such information, however, if used in conjunction with spirometric P-V information, may theoretically help elucidate actual consequences of PEEP application.

FRC measurements must be evaluated in conjunction with data regarding oxygenation as well as tidal compliance [33-36]. Although the latter relates inversely to the stiffness of the lung and/or chest wall, the tidal compliance traditionally used at the bedside does not necessarily track lung volume, as further increments of PEEP above a specific level may simply cause overdistension - indicated by accompanying increases of elastance [33]. Studies conducted in lung injury models have investigated the relationship between FRC and tidal compliance [33-35]. In a porcine oleic-acid-injury study, Rylander et al. [37] found that FRC was a more sensitive indicator of PEEP-induced aeration than was compliance. Additionally, Lambermont et al. [34] showed that FRC may potentially be useful in identifying an optimal PEEP level when it is associated with the best compliance and lowest dead space to tidal volume ratio [34]. There is still no irrefutable information regarding the range of values of FRC to be expected in the setting of ALI/ARDS. However, much of the available data strongly supports the potential use of FRC in therapeutic decision-making and its utility as a diagnostic tool. Perhaps relating FRC to its expected values is not as important as knowing the response of FRC to interventions or to the course of disease.

\section{Clinical implications of FRC measurement}

Important information can be extracted from the FRC value, because this measurement correlates with 'functional' (aerated and communicating) lung size [28]. Resting aerated lung volume is tightly correlated with oxygenation [36], estimated risk for VILI [15], work of breathing [38] and gas trapping [39]. As such, FRC could be used as an indicator of disease progression and response to therapy. Finally, FRC can also help monitor the relationship between body position changes and the physiological response of the compromised respiratory system [39]. Clinical experience shows that oxygenation is markedly affected by postural changes in certain patients. These hypoxemic episodes may be the result of position-related ventilation/perfusion changes associated with abrupt reductions in FRC or to regional perfusion changes [40,41].

Regarding the role of FRC in interpretation of lung mechanics, changes in lung volume could help 
characterize the nature and severity of lung disease. Resistance and compliance are expressed in absolute terms $\left(\mathrm{cm} \mathrm{H}_{2} \mathrm{O} / \mathrm{l} / \mathrm{s}\right.$ and $\mathrm{ml} / \mathrm{cm} \mathrm{H}_{2} \mathrm{O}$, respectively) that vary with aerated lung dimensions. Knowing the FRC value facilitates the separation of restrictive from obstructive disease and allows better interpretation of parenchymal gas exchanging efficiency [42]. Additionally, since 'specific compliance' and 'specific elastance' account for the resting size (volume) of the aerated lung, the response of the respiratory system to an imposed stress may be best evaluated when FRC is known [43]. By determining the size of the 'baby lung, FRC has the potential to elucidate the mechanical stress incurred during tidal breathing and the risk for VILI in the setting of ALI/ ARDS $[43,44]$. We must recognize that FRC values could lead to subject-specific interpretations of lung stress $\left(\mathrm{P}_{\mathrm{TP}}\right)$, and may be integral for assessing lung strain (tidal volume/FRC) - commonly equated with tissue 'stretch' [44]. With current techniques, valid FRC measurements can be obtained to calculate a strain ratio. The latter references the end-tidal volume to its resting level, with strain ratios exceeding 1.5-2.0 signaling concern for lung overstretch [44] (Fig. 1).

Similar principles relate to airway resistance. Whether in obstructive disease, ALI/ARDS, or other volumereduced states (e.g., surgical reduction of lung tissue, effusion-compressed lung), knowledge of FRC also enables calculation of specific resistance and provides better information regarding airway status [43, 44]. Additionally, non-symmetrical disorders of the chest wall (e.g., unilateral pleural effusion and increased IAP) may cause $P_{T P}$ and FRC to dissociate from each other [11]. Such dissociation may also be characteristic of some other lung disorders (e.g., secretion plugging, unilateral pneumonia, atelectasis, embolism, pneumothorax, etc.). In other words, separations or disconnections among these monitored mechanic variables, especially if trended, graphed and/or indexed, could be valuable in diagnosis and monitoring.

\section{Conclusion}

Calculating $\mathrm{P}_{\mathrm{TP}}$ based on Pes measurements and monitoring FRC are two complementary pieces of the diagnostic/monitoring puzzle to be added to traditional pulmonary mechanics stemming from $\mathrm{P}_{\mathrm{AW}}$ and tidal air flow. Mechanical ventilation guided by $\mathrm{P}_{\text {TP }}$ calculations opens possibilities for personalizing and improving the analysis of the mechanics of pulmonary injury. It seems clear that these newly available tools, used separately and/or together, have potential to improve delivery of respiratory care by characterizing the response to interventions or to the course of disease. Moreover, recognizable patterns and trends in correlated indexes of FRC and $\mathrm{P}_{\mathrm{TP}}$ in addition to traditional monitoring tools,

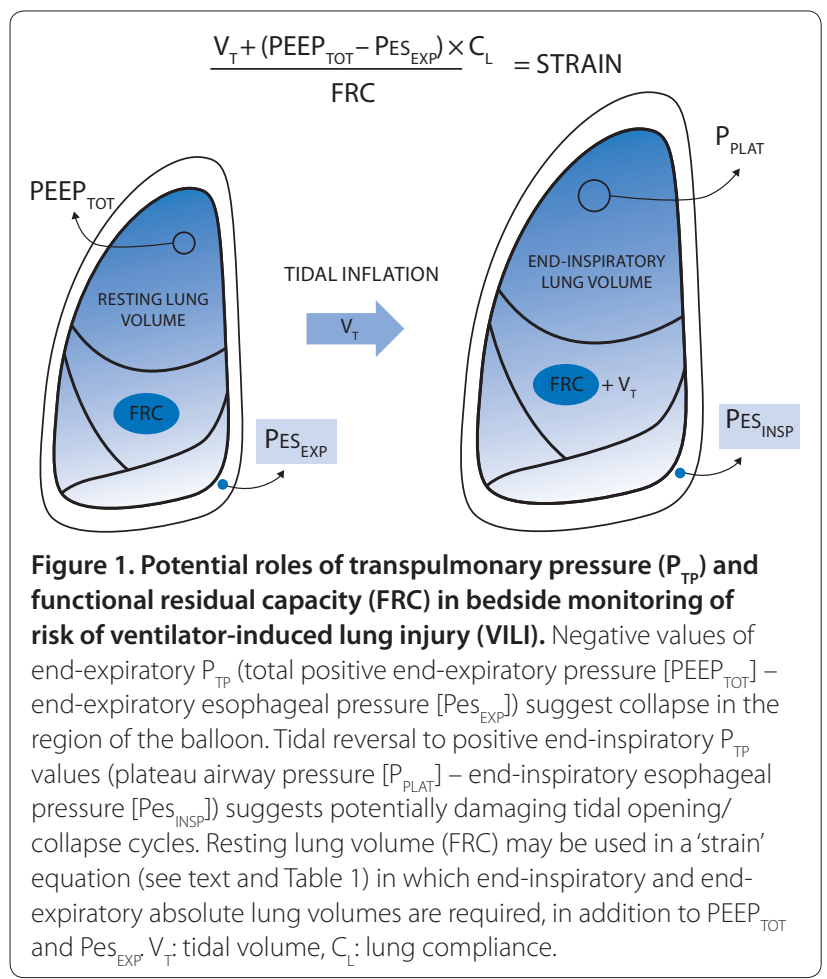

could help diagnose and/or provide an early warning to the clinician of impending danger in the settings of chest wall abnormalities (e.g., elevated IAP) and the asymmetrically distributed lung diseases often encountered in critical care. Instead of the first response being crisis intervention or expensive testing, earlier evaluation and prevention could be achieved by using and understanding FRC and $\mathrm{P}_{\mathrm{TP}}$ values. Computer technology already deployed should make such derived information easy to display.

Competing interests

GAC and JJM have received an investigator initiated grant from GE Healthcare.

\section{List of abbreviations used}

ALI: acute lung injury; ARDS: acute respiratory distress syndrome; BMI: body mass index; $C_{L}$ : lung compliance; ECMO: extracorporeal membrane oxygenation; FRC: functional residual capacity; IAP: intra-abdominal pressure; $\mathrm{P}_{\text {AW }}$ : airway pressure; PEEP: positive end-expiratory pressure; $\mathrm{PEEP}_{\mathrm{TOT}}$ : total positive end-expiratory pressure; Pes: esophageal pressure; Pes EXP. $_{\text {: }}$ endexpiratory esophageal pressure; Pes ${ }_{\mathbb{I N S P}}$ : end-inspiratory esophageal pressure; $P_{\text {PLAT: }}$ : plateau airway pressure; $P_{\text {TP: }}$ : transpulmonary pressure; VILI: ventilatorinduced lung injury; $V_{T}$ : tidal volume.

Published: 19 March 2013

\section{References}

1. Milic-Emili J, Mead J, Turner JM, Glauser EM: Improve technique for estimating pleural pressure from esophageal balloons. J Appl Physio/ 1964, 19:207-211.

2. Mead J, Gaensler EA: Esophageal and pleural pressures in man, upright and supine. J App/ Physio/ 1959, 14:81-83.

3. Pelosi P, Goldner M, McKibben A, Adams A, Eccher G, Caroni P, Losappio S, Gattinoni L, Marini JJ: Recruitment and derecruitment during acute respiratory failure: an experimental study. Am J Respir Crit Care Med 2001, 
$164: 122-130$

4. American Thoracic Society/European Respiratory Society: ATS/ERS Statement on Respiratory Muscle Testing. Am J Respir Crit Care Med 2002 166:518-624.

5. Baydur A, Behrakis PK, Zin WA, Jaeger M, Milic-Emili J: A simple method for assessing the validity of the esophageal balloon technique. Am Rev Respir Dis 1982, 126:788-791.

6. Talmor D, Sarge T, Malhotra A, O'Donnell CR, Ritz R, Lisbon A, Novack V, Loring $\mathrm{SH}$ : Mechanical ventilation guided by esophageal pressure in acute lung injury. N Engl J Med 2008, 359:2095-2104.

7. Agostoni $E$, Miserocchi G: Vertical gradient of transpulmonary pressure with active and artificial lung expansion. J App/ Physio/ 1970, 29:705-712.

8. Agostoni E, D'Angelo E, Bonanni MV: Topography of pleural surface pressure above resting volume in relaxed animals. J App/ Physio/ 1970 29:297-306.

9. Agostoni E, D'Angelo E, Bonanni MV: The effect of the abdomen on the vertical gradient of pleural surface pressure. Respir Physiol 1970, 8:332-346

10. Knowles JH, Hong SK, Rahn H: Possible errors using esophageal balloon in determination of pressure-volume characteristics of the lung and thoracic cage. J Appl Physiol 1959, 14:525-530.

11. Graf J, Formenti P, Santos A, Gard K, Adams A, Tashjian J, Dries D, Marini JJ: Pleural effusion complicates monitoring of respiratory mechanics. Crit Care Med 2011, 39:2294-2299.

12. Owens RL, Campana LM, Hess L, Eckert DJ, Loring SH, Malhotra A: Sitting and supine esophageal pressures in overweight and obese subjects. Obesity (Silver Spring) 2012, 20:2354-2360

13. Loring SH, O'Donnell CR, Behazin N, Malhotra A, Sarge T, Ritz R, NovackV, Talmor D: Esophageal pressures in acute lung injury: do they represent artifact or useful information about transpulmonary pressure, chest wall mechanics, and lung stress? J App/ Physio/ 2010, 108:515-522.

14. Talmor DS, Fessler HE: Are esophageal pressure measurements important in clinical decision-making in mechanically ventilated patients? Respir Care 2010, 55:162-172.

15. The Acute Respiratory Distress Syndrome Network: Ventilation with lower tidal volumes as compared with traditional tidal volumes for acute lung injury and the acute respiratory distress syndrome. N Engl J Med 2000 342:1301-1308.

16. Grasso S, Terragni P, Birocco A, Urbino R, Del Sorbo L, Fillipini C, Mascia L, Pesenti A, Zangrillo L, Ranieri VM: ECMO criteria for influenza A (H1N1)associated ARDS: role of transpulmonary pressure. Intensive Care Med 2012, 38:395-403.

17. Richard JC, Marini JJ: Transpulmonary pressure as a surrogate of plateau pressure for lung protective strategy: not perfect but more physiologic. Intensive Care Med 2012, 38:339-341.

18. Rimensberger PC, Bryan AC: Measurement of functional residual capacity in the critically ill. Relevance for the assessment of respiratory mechanics during mechanical ventilation. Intensive Care Med 1999, 25:540-542.

19. Pierson DJ: Measuring and monitoring lung volumes outside the pulmonary function laboratory. Respir Care 1990, 35:660-668.

20. Graf J, Santos A, Dries D, Adams AB, Marini JJ: Agreement between functional residual capacity estimated via automated gas dilution versus via computed tomography in a pleural effusion model. Respir Care 2010, 55:1464-1468.

21. Yernault JC, Pride N, Laszlo G: How the measurement of residual volume developed after Davy (1800). Eur Respir J 2000, 16:561-564

22. Suter PM, Schlobohm RM: Determination of functional residual capacity during mechanical ventilation. Anesthesiology 1974, 41:605-607.

23. Durig A: Über die Größe der Residualluft (About the size of the residual air). Zentralblatt Physiol 1903, 17:258-267.

24. Darling RC, Cournand A, Richards DW: Studies on the intrapulmonary mixture of gases. An open circuit method for measuring residual air. J Clin Invest 1940, 19:609-618.

25. Fretschner R, Deusch H, Weitnauer A, Brunner JX: A simple method to estimate functional residual capacity in mechanically ventilated patients. Intensive Care Med 1993, 19:372-376.

26. Chiumello D, Cressoni M, Chierichetti M, Tallarini F, Botticelli M, Berto V, Mietto C, Gattinoni L: Nitrogen washout/washin, helium dilution and computed tomography in the assessment of end expiratory lung volume. Crit Care 2008, 12:R150.

27. Brewer LM, Orr JA, Sherman MR, Fulcher EH, Markewitz BA: Measurement of functional residual capacity by modified multiple breath nitrogen washout for spontaneously breathing and mechanically ventilated patients. Br J Anaesth 2011, 107:796-805

28. Heinze $H$, Eichler W: Measurements of functional residual capacity during intensive care treatment: the technical aspects and its possible clinical applications. Acta Anaesthesio/ Scand 2009, 53:1121-1130.

29. Hedenstierna G: The recording of FRC - is it of importance and can it be made simple? Intensive Care Med 1993, 19:365-366.

30. Bikker IG, van Bommel J, Miranda DR, Bakker J, Gommers D: End-expiratory lung volume during mechanical ventilation: a comparison with reference values and the effect of positive end-expiratory pressure in intensive care unit patients with different lung conditions. Crit Care 2008, 12:R145.

31. Patroniti N, Saini M, Zanella A, Weismann D, Isgrò S, Bellani G, Foti G, Pesenti $\mathrm{A}$ : Measurement of end-expiratory lung volume by oxygen washinwashout in controlled and assisted mechanically ventilated patients. Intensive Care Med 2008, 34:2235-2240

32. Bikker IG, Scohy TV, Bogers A, Bakker J, Gommers D: Measurement of endexpiratory lung volume in intubated children without interruption of mechanical ventilation. Intensive Care Med 2009, 35:1749-1753.

33. Suter PM, Fairley HB, Isenberg MD: Effect of tidal volume and positive endexpiratory pressure on compliance during mechanical ventilation. Chest 1978, 73:158-162.

34. Lambermont B, Ghuysen A, Janssen N: Comparison of functional residual capacity and static compliance of the respiratory system during a positive end-expiratory pressure (PEEP) ramp procedure in an experimental model of acute respiratory distress syndrome. Crit Care 2008, 12:R91.

35. Maisch S, Reissmann H, Fuellekrug B: Compliance and dead space fraction indicate an optimal level of positive end-expiratory pressure after recruitment in anesthetized patients. Anesth Analg 2008, 106:175-181.

36. Heinze H, Sedemund-Adib B, Heringlake M, Meier T, Eichler W: Relationship between functional residual capacity, respiratory compliance, and oxygenation in patients ventilated after cardiac surgery. Respir Care 2010, 55:589-594

37. Rylander C, Hogman M, Perchiazzi G, Magnusson A, Hedenstierna G: Functional residual capacity and respiratory mechanics as indicators of aeration and collapse in experimental lung injury. Anesth Analg 2004, 98:782-789.

38. Marini JJ, Capps JS, Culver BH: The inspiratory work of breathing during assisted mechanical ventilation. Chest 1985, 87:612-618.

39. Marini JJ, Tyler ML, Hudson LD, Davis BS, Huseby JS: Influence of headdependent positions on lung volume and oxygen saturation in chronic air-flow obstruction. Am Rev Respir Dis 1984, 129:101-105.

40. Rodriguez-Nieto MJ, Peces-Barba G, Gonzalez Mangado N, Paiva M, Verbanck S: Similar ventilation distribution in normal subjects prone and supine during tidal breathing. J App/ Physio/ 2002, 92:622-666.

41. Behrakis PK, Baydur A, Jaeger MJ, Milic-Emili J: Lung mechanics in sitting and horizontal body positions. Chest 1983, 83:643-646.

42. Agustí A, Barnes PJ: Update in chronic obstructive pulmonary disease 2011. Am J Respir Crit Care Med 2012, 185:1171-1176.

43. Gattinoni L, Pesenti A: The concept of "baby lung". Intensive Care Med 2005, 31:776-784

44. Chiumello D, Carlesso E, Cadringher P, Caironi P, Valenza F, Cozzi P, Cressoni M, Colombo A, Marini JJ: Lung stress and strain during mechanical ventilation for acute respiratory distress syndrome. Am J Respir Crit Care Med 2008, 178:346-355.

doi:10.1186/cc12582

Cite this article as: Cortes GA, Marini JJ: Two steps forward in bedside monitoring of lung mechanics: transpulmonary pressure and lung volume. Critical Care 2013, 17:219. 